\title{
NOVEL SCHEME FOR PROTON AND HEAVY-ION ACCELERATORS
}

\author{
V. V. Gorev \\ RRC "Kurchatov Institute" and Institute of Physical Ballistics, 123182 Moscow, Russia
}

\section{INTRODUCTION}

Proton and heavy ion accelerators of the medial energy are very important in science and technology. First of all in the medicine, in the field of elimination of nuclear waste, for the creation safe subscritical reactors and so on. Their usage will help to cure cancer, to develop safety nuclear energetic, to protect environment and so on. Unfortunately large size of the accelerators that could be about $1 \mathrm{~km}$ length and high cost that is about 1 billion and even more gives limitation their wide applications. Right now the society understands the importance of all the problems listed above and we are able to create and develope new solution in the field of these accelerators design.

We have developed a conceptual design for highgradient linear proton and heavy ion medium energy accelerators, based on the new physical principle.

We are going to discuss only major ideas leaving all the details for the future examine.

\section{Accelerator scheme BESTAC (ballistical} electrostatical accelerator) has two parts. The main and additional, that are connected in one unit. The main part is responsible for the ion acceleration itself. It has quazispherical shape. The addition part is electron linac for which provides work ability of the main part.

BESTAC operates in impulse regime. The principle of BESTAC performance we can qualitatively explain by dividing the process of the impulse generation into 4 following stages:

1. Vacuum camera ("VC") is made from conductive materials, grounded and put into longitudinal magnetic field. VC has four main and few assisting vacuum windows. The main windows (\# 1,2,3,4) are located two perpendicular diameter of the chamber. A small specially shaped and constructed body is injected into VC at the initial moment throughout the window \# 1 . This body (so called "ballistic anode") freely moves from window \# 1 to window \# 2 along ballistic trajectory and crosses the central part of $\mathrm{VC}$.

2. Ballistical anode is irradiated by positron beam than it cross the central part of vacuum chamber. We should have such energy current duration and form of the beam impulse to provide accumulation of the positive charge on the ballistical anode, but not the scattering and losses of the positrons on ballistical anode and ballistical anode heating. This is regime exists according to our calculations and is called "change pumping". Quazistatical difference of potential appears between ballistical anode and vacuum chamber wall while ballistical anode moved along ballistic trajectory. The quantity and duration of the potential is determined by competition between the processes of the charge accumulation of positrons and charge losses.

The loss of the charge caused by numerous reasons (cold ion emission from the surface of ballistic anode, cold electron emission from the inner surface of vacuum chamber, photoeffects, electrical break of physical vacuum, generation of electron-positron cascade and so on. The difference of potential between ballistical anode and vacuum chamber could be very high if we can produce sufficient current of positrons, sufficient size of vacuum chamber and sufficiently high vacuum. Current is the most important feature. The potential difference is possible to use for the ions acceleration during the time of its existence.

3. The surface of ballistical anode itself can be the source of the protons and heavy ions, if it is radiated by laser impulse and transformed into plasma. The second way for the protons and heavy ions emission can be the special construction of ballistical anode, when the electric field strength on the small part of ballistical anode surface that is located in front of the window \# 2 is enough for cold ion emission. This is very similar to the ion source, based on field evaporation. If our goal is the production of neutral particles we have to put the source of negative ions on the line of windows \# 2- \# 4 out of vacuum chamber. In this case at first the negative ions are accelerated from the wall of vacuum chamber towards ballistical anode. On ballistical anode they are neutralized and then leave vacuum chamber through the window \# 4. They can be transformed into positive ions if the thickness of ballistical anode is large enough and additionally accelerated between ballistical anode and vacuum chamber similar the tandem acceleration scheme.

4. Ballistical anode continues to move uncharged along ballistic trajectory, if the process of ballistical anode 
charge and discharge occurs during ballistical anode flight time across central part of vacuum chamber. Then it leave vacuum chamber through the window \# 3 .

It is necessary to repeat all these cycles 1-4 to generate impulse of protons and ions current again.

Addition part of BESTAC is consists of two parts: the accelerator of the positrons (linac) and the source of positrons.

Accelerator shapes the positron beam for the ballistical anode irradiation and charge.

The electric field between ballistical anode and vacuum chamber is the energy source for the ion beam.

This field receives energy from the kinetic energy of the positron beam. We can say that the specially constructed ballistical anode is the transformer of the light positive particles into the heavy ones. Positrons penetrates into ballistical anode substance, and can thermolized and annihilated if their energy is not very high. The part of the electrons disappears and quazineutrality breaks. The major part of electric field is located in the space between ballistical anode and vacuum chamber, and the rest penetrates into ballistical anode substance up to few interatomic distances.

3. The charge pumping regime is not normal for beamtarget interaction. As usual the ballistical anode substances is heated and transformed into plasma. In this case it is impossible to have the regime of charge pumping because even cold plasma has practically unlimited emission capability. To realize the maximum charge pumping regime it is necessary to fulfil few conditions.

The major of them are:

1. Ballistical anode has to be positive; In this case the electric field strength on ballistical anode surface can be up to $10^{\wedge} 9 \mathrm{~V} / \mathrm{cm}$ and its potential $10^{\wedge} 9 \mathrm{r}(\mathrm{cm})$ volts.

2. It is necessary to use the light charged particles because they have the penetration length that is two orders higher than ions have and as a result of the ballistical anode heating will be two orders less.

3. The positrons energy has to grow in time according to the linear law if the beam current is constant ("saw toothed "voltage). The quantity of electric charge and potential on ballistical anode will have a linear increase also at the constant positrone current. To overcome this potential we should have the linear increase of kinetic energy of the positrones also, according to saw toothlaw and positrones energy should be a little bit higher $(\Delta \varepsilon)$ than electrical potential energy of the ballistocal anode surface.

4. The difference between kinetic energy of the particles and the quantity of energetic width $\Delta \varepsilon$ is limited $\Delta \varepsilon$ is determined by the target size, because length of the positrone penertration has to be lower than target size. In this case the positrone penetrates into the substance ballistical anode, thermolized and annihilates approximatly homogeneously in the ballistical anode volume. this reduces the local overheating.

5. Geometrical shape of ballistical anode should provide the maximum cross section of the positrone neglection at the determined value of $\Delta \varepsilon$. We can guarantee almost no loss of positrons through scattering in the the longitudianl magnetic field.

We should have sufficiently high vacuum and large vacuum chamber size to quarantee the regime of charge pumping. The residual gas is going to be tunnel ionized in the vacuum chamber by the strong electric field that leads the electric current formation from vacuum to ballistical anode and ion current formation on the inner surface of vacuum chamber. Definitly this current will be increased with the pressure of residual gas increasee. Gas ionization is going into the sphere with radius equal to few ballistical anode scale during charge pumping.

4. Let us carry out main estimations for the case of 1 $\mathrm{GeV}$ proton accelerator by assuming that electrical field strenth is about $5 \cdot 10^{\wedge} 8 \mathrm{~V} / \mathrm{cm}$, one obtains:

1. Radius of ballistical anode $-2 \mathrm{~cm}$

2. Radius of vacuum chamber $-200 \mathrm{~cm}$

3. Number of positrons $\sim 10^{\wedge} 15-10^{\wedge} 16$

4. Velocity of ballistical anode $-4 \mathrm{~m} / \mathrm{sec}(\mathrm{min})-1000$ $\mathrm{m} / \mathrm{sec}(\max )$

5. Linac positron current - $\left(10^{\wedge}-2-25\right)$ A

6. Saw-tooth low: $\gamma \mathrm{mc}^{\wedge} 2(\mathrm{Gev})=$

$$
\begin{aligned}
& \frac{t}{\tau}+\Delta \varepsilon \\
& \tau=10^{\wedge}-2(\max )-4.10^{\wedge}-5(\min ) \\
& \text { 7. } \Delta \varepsilon \sim 20 \mathrm{MeV} \text { (for light materials), } \\
& \sim 80 \mathrm{MeV} \text { (for medial materials) } \\
& \sim 160 \mathrm{MeV} \text { (for heavy materials) } \\
& \text { 8. } \frac{\Delta \varepsilon}{\gamma m c^{2}} \sim(2-16) \% \\
& \text { 9. Vacuum } \sim 10^{\wedge}(-11-12) \text { torr }
\end{aligned}
$$

\section{CONCLUSIONS}

We propose new physical principle for the generation of high intensive impulses of electrical fields and for acceleration of protons and ions. The principle involves:

1.Usage of the ballistical anode, as a transformer of charge particles.

2.Usage of the positron beams for the charge pumping of ballistical anode.

3. Usage of the high vacuum and spherical geometry as a high voltage insulator.

4. Usage of the high gradient linac as an energy source for protons and ion beams. 